\title{
Reuse-based Navigation Application Modeling Methodology
}

\author{
Sangyoung Lee \\ Department of Public Health Administration, Namseoul University, South Korea \\ sylee@nsu.ac.kr
}

\begin{abstract}
The recent projects for GNSS component extraction and development process extract system components by extracted use case after requirement analysis to extract business components. Then, business components extracted by an interaction diagram are refined, and the components to be included in the system components that are extracted for defining architecture are found and combined. However, the method has an advantage in that its extraction clearly separate system and business components because it clearly defines the system component that can be functionally reused in respect of system service. On the other hand, the method has disadvantages in that it is hard to discern whether the word in the type diagram has a meaning or not by a work-site operator who has no systemic concept and it is difficult to draw a relation between those words when the operator draws a type diagram to figure out business requirements. In this paper suggests a modeling methodology to build a key business processoriented system based on extraction of key components that is not limited to a certain domain. From this business-oriented perspective, major key components are extracted and applied to relevant application, which contributes to improved software quality such as development, maintenance, productivity, etc. To extract components, necessary functions for a system are classified functionally and hierarchically and modularized. From the process, the self-execution unit is produced for a meaningful service. It does not use the existing procedure- or object-oriented system, a process-oriented component design method, but business-based modeling methodology, and it suggests a process to extract, define, and analyze components that are commonly used in relevant application domains which is a foundation for building GPS system.
\end{abstract}

Keywords: GNSS, GPS, reuse, modelling. 


\section{Introduction}

GPS (Global Positioning System) is invented for the military and developed by the Pentagon, but it is available for civilian use after opening the civil codes. GNSS includes US GPS, European Galileo, etc (Lau and Cross, 2007; Jacob et al., 2009; Duca et al.,2009). In GNSS fields, developed countries such as the United States, Europe, and other countries are dedicated to secure the original technologies, including GNSS simulator, etc. that are available for trail test and evaluation. Also, the technology is widely used for the military uses such as precise measurement, but also for civilian uses such as car navigation system, small lightweight GPS receiver for hiking

The principle of GNSS is to measure the propagation time that is considered to be the time from the satellite to the receive, calculate distance from satellite to receiver, and measure the current location using trigonometry (Guerrier, 2009; Glass, 2006; Buschmann et al., 2007). In these applications, the focus is that they are located on different domain, but it has common and key components (Waegli, 2012; Lappalainen, 2005). In other word, the existing applications have disadvantages in terms of separate implementation and development for each domain even though they have common components (Lockwood, 2012; Fisher and Kunches, 2011). Thus, from business-oriented viewpoint, GNSS applications extract key components, which is utilized for building related applications. And, their software quality has to be improved in terms of development, maintenance, and productivity. In short, the GNSS component will have to be made of the self-execution unit that hierarchically modularizes functions provided by a system. By applying the reusable property, the component is developed by distinguishing between reusable and new one, which reduces its development time and secures quality component. To extract quality one, a systematic modeling method that accurately expresses user's demands is required (Wernik et al., 2003).

Business-oriented viewpoint, GNSS applications extract key components, which is utilized for building related applications. And, their software quality has to be improved in terms of development, maintenance, and productivity. In short, the GNSS component will have to be made of the self-execution unit that hierarchically modularizes functions provided by a system. By applying the reusable property, the component is developed by distinguishing between reusable and new one, which reduces its development time and secures quality component. To extract quality one, a systematic modeling method that accurately expresses user's demands is required For development of GPS system from a business perspective, this paper aims to extract key components that is not limited to the specific domain and suggest a modeling method that is able to build business process-oriented system. 


\section{Related Works}

By ensuring reusability and independence of software module, the component shows the new paradigm to solve problems of complexity and productivity of software. Various methodologies of developing UML - key methodology based on component - have been actively used in diverse fields, including development process, system engineering, and business process (Cannon et al., 2003; Hapgood and Thomson, 2010; Flynn, 2001; Spogli, 2009).

Recent projects for GNSS component development process extracted components after requirement analysis of business components (Spogli et al., 2010; Alfonsi et al., 2011; Ruiu et al., 2012). Then, business components extracted by an interaction diagram are refined, and the components to be included in the system components that are extracted for defining architecture are found and combined (Bougard et al., 2011; Doherty et al., 2000; Liu and Lachapelle, 2002; Peng, 2016). However, the method has an advantage in that its extraction clearly separate system and business components because it clearly defines the system component that can be functionally reused in respect of system service (Byun, 2017; Balaji and Dakshayini, 2018). On the other hand, the method has disadvantages in that it is hard to discern whether the word in the type diagram has a meaning or not by a work-site operator who has no systemic concept and it is difficult to draw a relation between those words when the operator draws a type diagram to figure out business requirements (Kang and Chung, 2017; Bashar, 2015).

In this paper, it does not use the existing procedure or object-oriented system, a process-oriented component design method, but business process-based modeling methodology, and it suggests a process to extract, define, and analyze components that are commonly used in relevant application domains which is a foundation for building GPS system to overcome these disadvantages.

\section{GPS System Analysis}

The GNSS consist of three main satellite technologies: GPS, Glonass and Galileo. Each of them consists mainly of three segments: (a) space segment, (b) control segment and (c) user segment. These segments are almost similar in the three satellite technologies, which are all together make up the GNSS. As of today, the complete s next few atellite technology is the GPS technology and most of the existing worldwide applications related to the GPS technology. The GNSS technology will become clearer after the operation of Galileo and the reconstruction of Glonass in the years.

Global Navigation Satellite Systems (GNSS) technology has become vital to many applications that range from city planning engineering and zoning to military applications. It has been widely accepted globally by governments and organizations. That is why we expect to have very soon at least three GNSS systems: the USA GPS, European Galileo, and the Russian Glonass systems. There is a 
multibillion dollar investment in this field and intensive worldwide research activities. The impressive progress in wireless communications and networks has played a great role in increasing interest in GNSS and providing enabling methodologies and mechanisms. It is expected that all $3 \mathrm{G}$ and future generations of cellular phones will be equipped with GNSS chips. GNSS technology dominates the outdoor navigation, which provides accuracy to the range of few meters to $10 \mathrm{~m}$ in single point positioning technique or sub-meter to a few meter level in differential GNSS technique (DGNSS). Different techniques have been developed recently for indoor positioning. They offer either absolute or relative positioning capabilities with acceptable precision. Combining these technologies with GNSS allows to provide a more reliable and robust location solution. Most common implementation of Hybrid technology for GSM, GPRS and WCDMA is to combine A-GNSS with Cell-ID.

Analysis of business requirements and extraction of business class models are required prior to developing an application. According to these requirements, mobile and reference stations receive signals from GPS satellite. The satellite continuously transmits information about time, its orbit, rough orbits of entire satellites, their reliability, and error correction factors that are required for other navigation system, and the receivers of mobile and reference stations continuously receive these message signals for navigation.

The receiver of mobile station receives signals from GPS satellite and processes data. And then, using the signal received from the satellite, the location of mobile station is measured, orbital information relating the satellite is extracted, and rough information of orbit such as the number of satellites and the satellite number that is used is extracted. In addition, the receiver extracts the time to receive signals from the GPS satellite. The mobile station transmits processed data to the control station, including its location, satellite number, the number of satellites, time to receive signal from the satellite, location of other satellites, and speed that is calculated with speedometer in the mobile station. It also transmits its own unique ID, information and its condition to the control station

Furthermore, the reference station calculates error based on the signal from GPS satellite and correction value of each satellite after comparing signal from the GPS satellite with the received signal, by using precisely known value of location of the reference station. And, the reference station transmits the calculated error in RTCM format to the control station.

The major business class extracts candidates from the requirement documents using the noun phrase approach that is an approach to find the corresponding noun in the requirement document, and all nouns are considered as candidates in the approach. The candidate list is classified into three main types: irrelevant class, relevant class, and fuzzy class.

Extracted candidates are divided into relevant class and fuzzy class. The initial 
business class model is shown in Figure 1, which is a class diagram of extracted candidates (Lee, 2019).

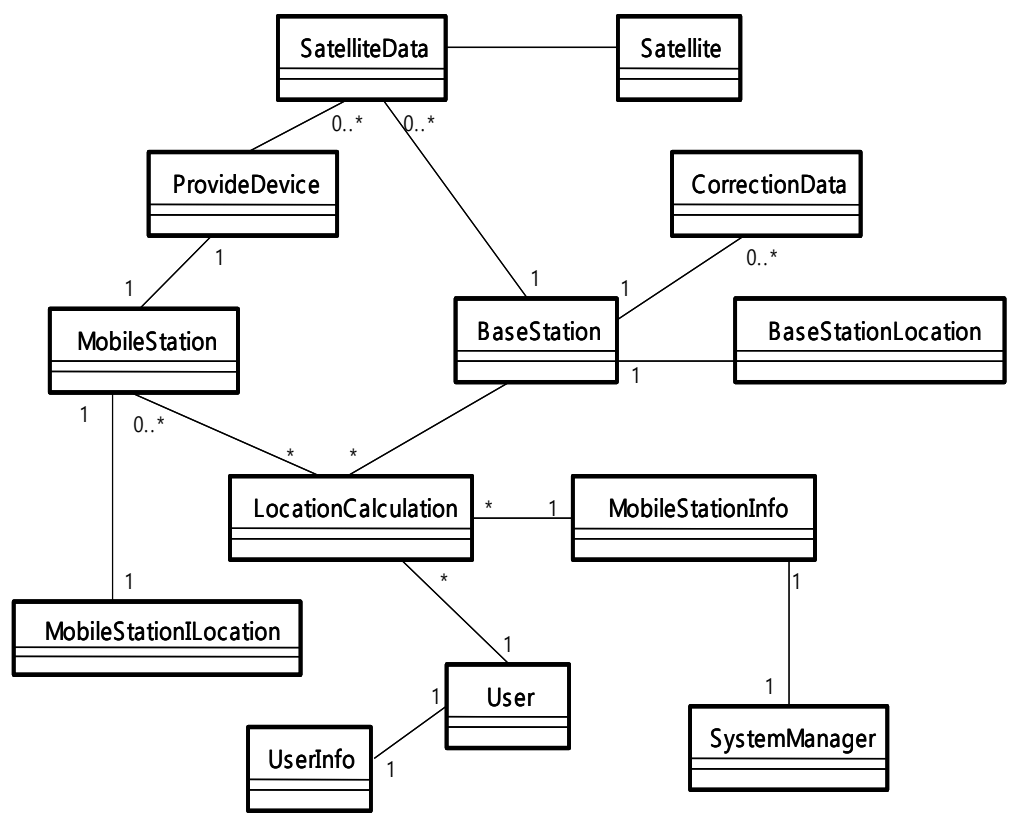

Fig. 1: Initial business class model

\section{GPS System Modelling Based on Business}

Hou In GNSS fields, developed countries such as the United States, Europe, and other countries are dedicated to securing the original technologies, including GNSS simulator, etc. that are available for trail test and evaluation. Also, the technology is widely used for the military uses such as precise measurement, but also for civilian uses such as car navigation system, small lightweight GPS receiver for hiking.

The extraction rule of major use cases is as follows. Major use cases only related to success of a scenario. If an exception occurred while processing the use case scenario and the use cases that do not concerns flows, the use cases are not regarded as a successful use case. A use case diagram is usually simple. It does not show the detail of the use cases:

- It only summarizes some of the relationships between use cases, actors, and systems.

- It does not show the order in which steps are performed to achieve the goals of each use case.

As said, a use case diagram should be simple and contains only a few shapes. If yours contain more than 20 use cases, you are probably misusing use case diagram. The figure below shows the UML diagram hierarchy and the positioning of the UML Use Case Diagram. As you can see, use case diagrams belong to the family of 
behavioral diagrams. Use cases represent only the functional requirements of a system. Other requirements such as business rules, quality of service requirements, and implementation constraints must be represented separately, again, with other UML diagrams. Identifying the Use Cases, and then the scenario-based elicitation process carries on by asking what externally visible, observable value that each actor desires. The following questions can be asked to identify use cases, once your actors have been identified

From the relevant business process perspective, scenario are as follows.

- The receivers of mobile and reference stations continuously receive the navigation message signals, which is information about time, its own orbit, rough orbits of entire satellites, their reliability, and error correction factors that are required for other navigation system.

Extensions: If the receiver does not get a signal, it will be an error. It prepares to receive signals by back into the situation that can receive the signal.

- Using the signal from GPS satellite, the location of mobile station is measured, orbital information relating the satellite is extracted, and rough information of orbit such as the number of satellites and the satellite number that is used is extracted. In addition, the receiver extracts the time to receive signals from the GPS satellite.

Extensions: If the receiver does not process the data, it receives the signal from the satellite again and processes the data.

- The mobile station transmits processed data to the control station, including its location, satellite number, the number of satellites, time to receive signal from the satellite, location of other satellites, and speed that is calculated with speedometer in the mobile station. It also transmits its own unique ID, information and its condition to the control station.

Extensions: If transmission is failed, it transmits data again.

- The reference station calculates the correction value of each satellite after comparing signal with the received signal, by using precisely known value of location of the reference station, and the results are exported into RTCM (Radio Technical Commission for Maritime Services) standard.

- The reference station calculates the correction value of each satellite after comparing signal with the received signal, by using precisely known value of location of the reference station. It transmits the calculated RTCM error information to the control station. At this time, all error information is transmitted.

Extensions: If transmission is failed, it transmits data again.

- The control station calculates the correct location of the mobile station by using the correction value in RTCM format obtained from the reference station and information about location and satellite obtained from the mobile station. 
Extensions: If the control station does not measure the location of mobile station, it sends an error message and obtain information again.

At first, the range of the business class model is determined. As shown in Figure 2, delete the class that has no relation and decide the range of the model (Lee, 2019).

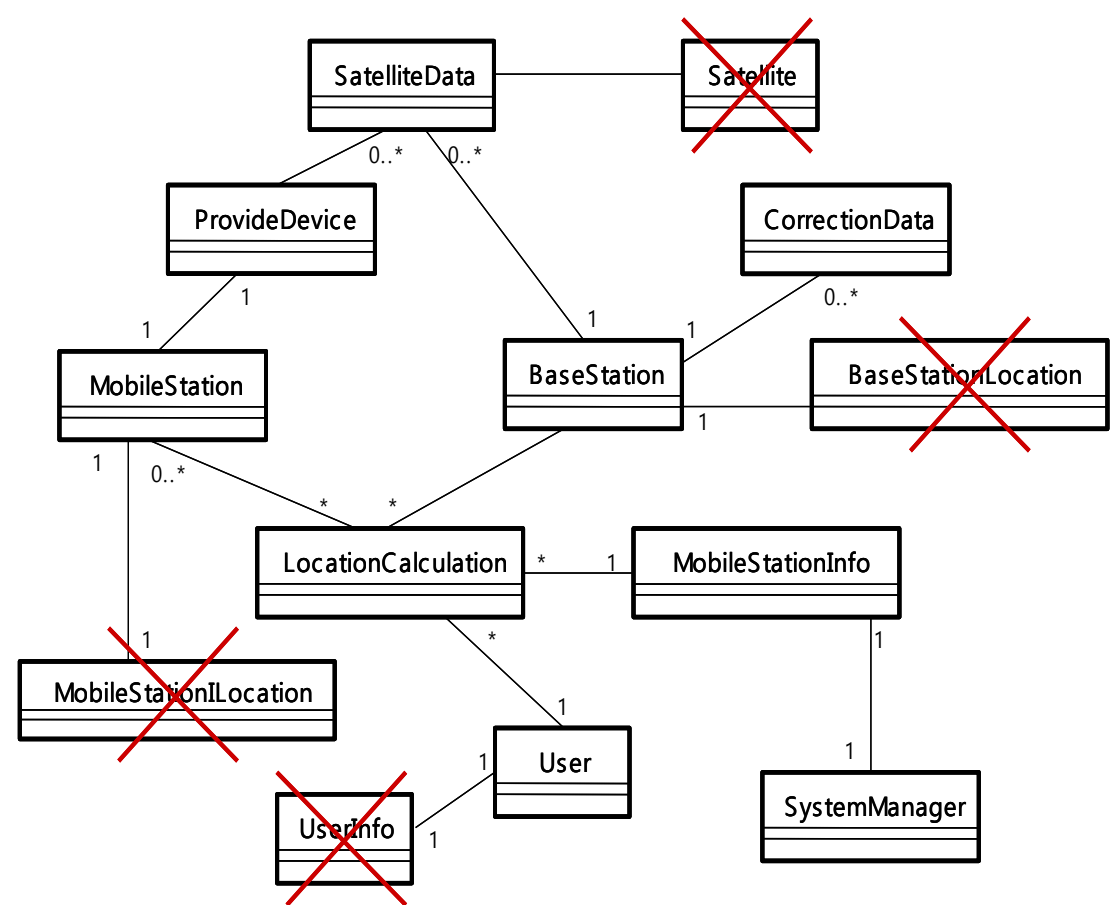

Fig. 2: Determine business class model scope

The business class model that refines the initial business class model is shown in Figure 3(Lee, 2019).

- The receivers of mobile and reference stations receive signals from the GPS satellite. The signal contains information about time, its own orbit, rough orbits of entire satellites, their reliability, and error correction factors that are required for other navigation system.

- The receiver of mobile station receives signals from GPS satellite and processes data. And then, using the signal, the location of mobile station is measured, orbital information relating the satellite is extracted, and rough information of orbit such as the number of satellites and the satellite number that is used is extracted. In addition, the receiver extracts the time to receive signals from the GPS satellite.

- The mobile station transmits processed data to the control station, including its location, satellite number, the number of satellites, time to receive signal 
from the satellite, location of other satellites, and speed that is calculated with speedometer in the mobile station. It also transmits its own unique ID, information and its condition to the control station.

- The reference station calculates error based on the signal from GPS satellite. The calculated results are exported to RTCM format

- The reference station transmits the correction value of each satellite in RTCM format.

- The location of mobile station is calculated by using the correction value in RTCM format obtained from the reference station and information about location and satellite obtained from the mobile.

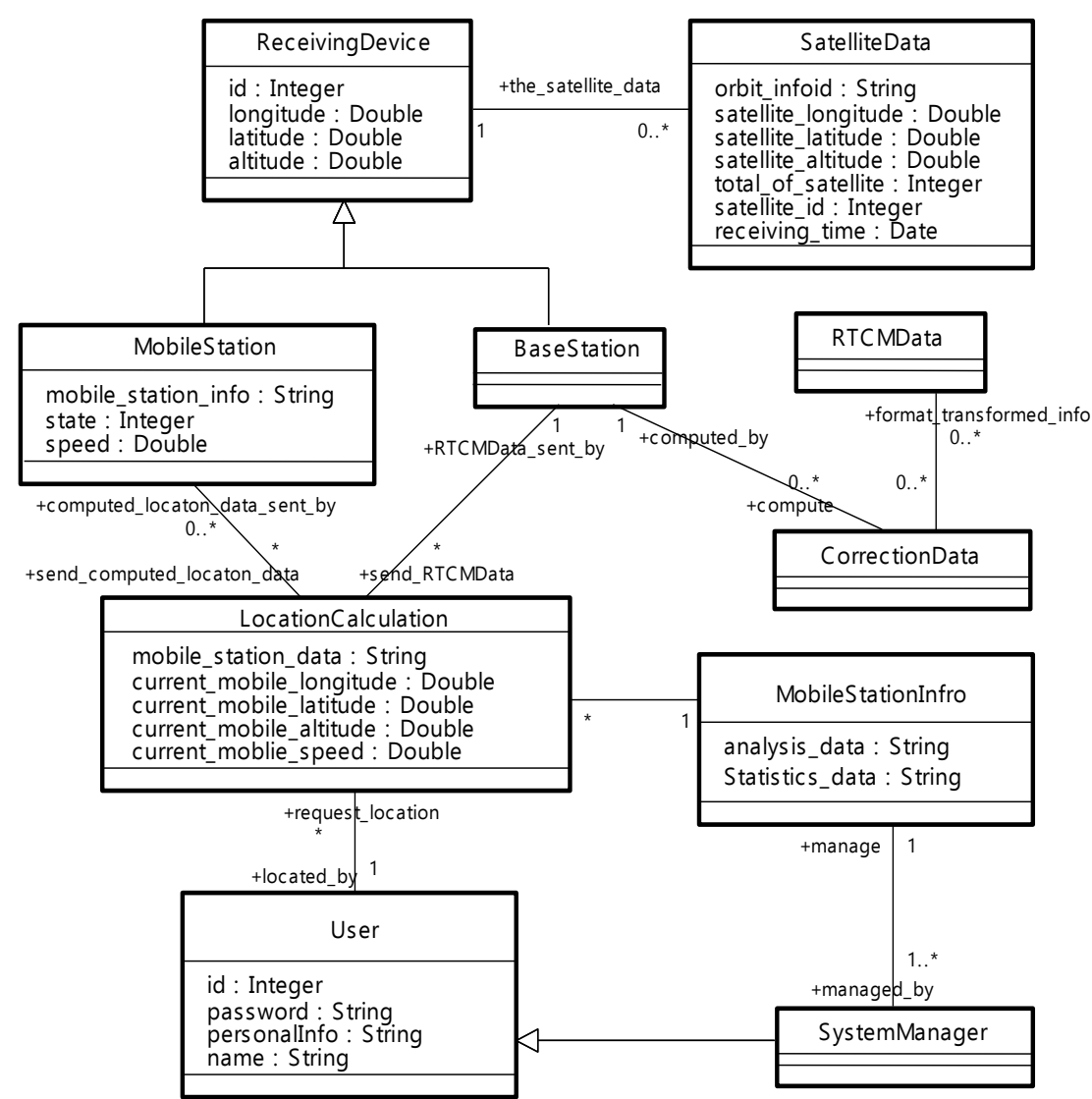

Fig. 3: Business class model

As shown in Figure 4, extracted stereotype of key classes is expressed as <<core>> to indicate the business class model. The diagram that represents relationship between extracted key classes and corresponding interface. 


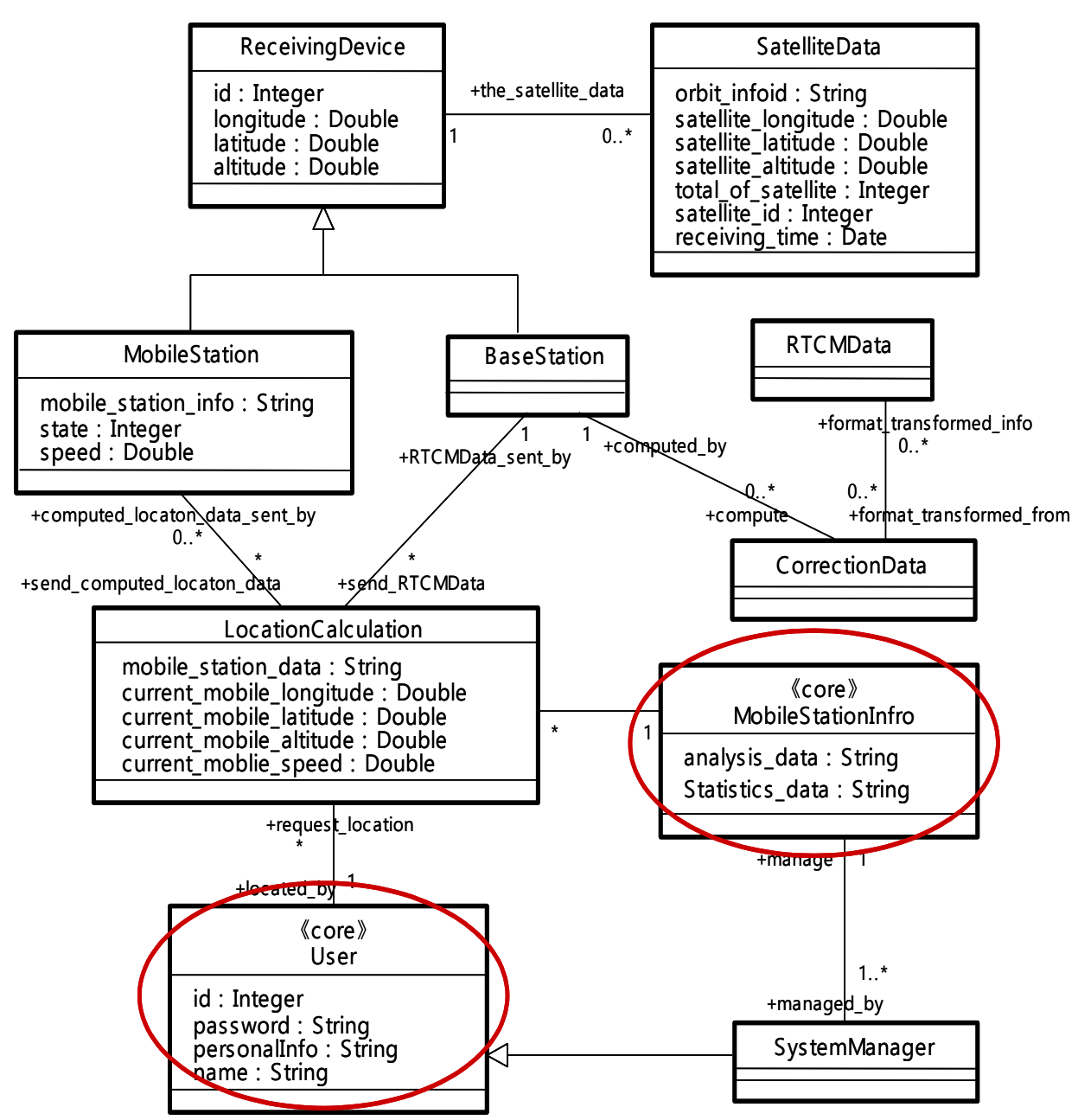

Fig. 4: Business class model representing the extracted core classes

\section{Conclusion}

The principle of GNSS is to measure the propagation time that is considered to be the time from the satellite to the receive, calculate distance from satellite to receiver, and measure the current location using trigonometry. In these applications, the focus is that they are located on different domain, but it has common and key components. In other word, the existing applications have disadvantages in terms of separate implementation and development for each domain even though they have common components. Thus, from business-oriented viewpoint, GNSS applications extract key components, which is utilized for building related applications. And, their software quality has to be improved in terms of development, maintenance, and productivity. In short, the GNSS component will have to be made of the selfexecution unit that hierarchically modularizes functions provided by a system. By applying the reusable property, the component is developed by distinguishing 
between reusable and new one, which reduces its development time and secures quality component. To extract quality one, a systematic modeling method that accurately expresses user's demands is required.

Especially, the recent projects for GNSS component extraction and development process extract system components by extracted use case after requirement analysis to extract business components. Then, business components extracted by an interaction diagram are refined, and the components to be included in the system components that are extracted for defining architecture are found and combined. However, the method has an advantage in that its extraction clearly separate system and business components because it clearly defines the system component that can be functionally reused in respect of system service. On the other hand, the method has disadvantages in that it is hard to discern whether the word in the type diagram has a meaning or not by a work-site operator who has no systemic concept and it is difficult to draw a relation between those words when the operator draws a type diagram to figure out business requirements.

In this paper, it does not use the existing procedure- or object-oriented system, a process-oriented component design method, but business process-based modeling methodology, and it suggests a process to extract, define, and analyze components that are commonly used in relevant application domains which is a foundation for building GPS system to overcome these disadvantages. To develop GPS system from the business perspective, this paper suggests a modeling methodology to build a key business process-oriented system based on extraction of key components that is not limited to a certain domain. Although there are disadvantages of the existing development methods of application in that there are common modules between domains, it makes up for in disadvantages, which is implementing and developing functions in the separate domain due to ease of development. From this businessoriented perspective, major key components are extracted and applied to relevant application, which contributes to improved software quality such as development, maintenance, productivity, etc. To extract components, necessary functions for a system are classified functionally and hierarchically and modularized. From the process, the self-execution unit is produced for a meaningful service. This study suggests a modeling method, which is that the component is developed by distinguishing between reusable and new one by applying the reusable property, which reduces its development time and secures quality component. It does not use the existing procedure- or object-oriented system, a process-oriented component design method, but business-based modeling methodology, and it suggests a process to extract, define, and analyze components that are commonly used in relevant application domains which is a foundation for building GPS system.

\section{Acknowledgements}

Funding for this paper was provided by Namseoul university. 


\section{References}

Alfonsi, L., L. Spogli, G. De Franceschi, V. Romano, M. Aquino, A. Dodson, and C. N.Mitchell (2011). Bipolar climatology of GPS ionospheric scintillation at solar minimum. Radio Science., 46.

Abul Bashar. (2015). Simulation Frameworks to Assess QoS Provisioning Solutions for Cloud-based Services. International Journal of Private Cloud Computing Environment and Management, 2 (2), 27-42.

Bougard B., J-M. Sleewaegen, L. Spogli, Sreeja V. V., J. F. (2011). Galera Monico, CIGALA: Challenging the Solar Maximum in Brazil with PolaRxS, Proceeding of the ION GNSS 2011, Portland, Oregon.

Balaji Prabhu B V, Dakshayini M. (2018). An Efficient Data Analytics-Cloud Integrated Decision Support Service for Smart Agriculture. International Journal of Internet of Things and Big Data, 3 (2), 1-10.

Cannon, P., Angling, M., Barclay, L., Curry, C., Dyer, C., Edwards, R., Greene, G., Hapgood, M., Horne, R., Jackson, D., Mitchell, C.N., Owen, J., Richards, R., Rogers, C., Ryden, K., Saunders, S., Sweeting, M., Tanner, R., Thomson, A., Underwood, C., (2003). Extreme space weather: impacts on engineered systems and infrastructure. Royal Academy of Engineering.

Committee on the Societal and Economic Impacts of Severe Space Weather Events: A Workshop, Severe Space Weather Events understanding societal and economic impacts. The National Academies Press, Washington, D.C., 2008,

China Satellite Navigation Office. (2010). BeiDou (COMPASS) Navigation ion Satellite System Development. Munich Satellite Navigation.

Doherty, P.H., Delay, S.H., Valladares, C.E., Klobuchar, J., (2000). Ionospheric scintillation effects in the equatorial and auroral regions. In: Proceedings of ION GPS 2000, Salt Lake City, USA.

Flynn, D. (2001). Space Weather Market Analysis: Summary Report for the ESA Space Weather Working Team. (E. N. 14069/99/NL/SB, Editor, Astrium, Producer, \& Hetfordshire, England)

Frank Buschmann, Kevlin Henney, Douglas C. Schmidt. (2007). Pattern-oriented Software Architecture: On Patterns and Pattern Languages. John Wiley and Sons. 
Fisher, G., and J. Kunches. (2011), Building resilience of the Global Positioning System to space weather. Space Weather, 9.

G. Del Duca, R. Perago, V. Paciucci, G. Di Bitonto, and F. Principe. (2009). "Verification of GNSS Applications at Italian Regional Airports", Proc. of ENCGNSS. Naples (Italy), May, 3-6.

Guerrier, S. (2009). "Improving Accuracy with Multiple Sensors: Study of Redundant MEMS-IMU/GPS Configurations". Proceedings of the ION GNSS 2009, Savannah, GE, USA.

Hapgood, M and A Thomson. (2010). Space weather: Its impact on Earth and implications for business. Lloyd's 360 Risk Insight. London, UK.

Liu G., and Lachapelle G. (2002). Ionosphere Weighted GPS Cycle Ambiguity Resolution, Proceedings of the U.S. Institute of Navigation National Technical Meeting, (San Diego, CA, January 2002), 889-899.

Lappalainen, H., K. Kauristie, and R. Pirjola. (2005). Space weather and risk management. Advances in Geosciences, 3, 23-27.

L. Lau, P. Cross. (2007). "Development and testing of a new ray-tracing approach to GNSS carrier-phase multipath modelling”. Journal of Geodesy, 81 (11), 713-732.

Lockwood, M., M. J. Owens, L. Barnard, C. J. Davis, and S. Thomas. (2012), Solar Cycle 24: what is the Sun up to? Astronomy and Geophysics, 53 (3), 9-3.

M.Jacob, M., S. Schön, U. Weinbach and T.Kürner. (2009). "Ray Tracing Supported Precision Evaluation for GPS Indoor Positioning." In Proc. 6th Workshop on Positioning. Navigation and Communication (WPNC), 15-22.

Min Wook Kang and Yun Won Chung. (2017). A Novel Power Saving Scheme for Base Stations in 5G Network. International Journal of Mobile Device Engineering, 1(1), 27-34.

O. Terzo, P. Ruiu, L. Mossucca, M. A. Francavilla and F. Vipiana. (2012). Grid Infrastructure for Domain Decomposition Methods in Computational ElectroMagnetics. Grid Computing - Technology and Applications, Widespread Coverage and New Horizons, Soha Maad (Ed.), InTech, 247-266.

Peng Hai-yun. (2016). Energy Consumption of Wireless Sensor Network Research Problem. International Journal of Internet of Things and Big Data. 1 (1), 29-36. 
Robert L. Glass. (2006). The Standish report: does it really describe a software crisis? Communications of the ACM, 49 (8), 15-16.

Spogli, L.; Alfonsi, L.; De Franceschi, G.; Romano, V.; Aquino, M. H. O.; Dodson, A. (2009). Climatology of GPS ionospheric scintillations over high and mid-latitude European regions, Ann. Geophys., 27.

Spogli, L.; Alfonsi, L.; De Franceschi, G.; Romano, V.; Aquino, M. H. O.; Dodson, A. (2010). Climatology of GNSS ionospheric scintillations at high and mid latitudes under different solar activity conditions. Il Nuovo Cimento B.

Siwoo Byun. (2017). Design of Efficient Index Management for Column-based Big Databases. International Journal of Internet of Things and Big Data. 2 (1), 23-28.

Sang Young Lee. (2019). Reuse-based Navigation Application Modeling Methodology, World Journal of Wireless Devices and Engineering. GLOBAL VISION PRESS, 3 (1), 9-14.

Wernik, A. W., Secan, J. A., and Fremouw. E. J. (2003). Ionospheric irregularities and scintillation. Advances in Space Research, 31(4).

Waegli, A., et al. (2012). Noise reduction and estimation in multipleelectro mechanical inertial systems. Measurement Science and Technology, 21. 